\title{
Bulk and Local Magnetic Susceptibility of $\mathrm{ErB}_{12}$
}

A. $\mathrm{Bogach}^{a}, \mathrm{~L} . \mathrm{Bogomolov}^{a}, \mathrm{~V} . \mathrm{Glushkov}^{a}, \mathrm{~S} \mathrm{DemisheV}^{a}$, D. Sluchanko ${ }^{a}$, N. Sluchanko ${ }^{a, *}$, N. Shitsevalova ${ }^{b}$, A. LevChenKo ${ }^{b}$, V. Filipov ${ }^{b}$, K. FlachBarT ${ }^{c}$ AND

K. Siemensmeyer ${ }^{d}$

${ }^{a}$ A.M. Prokhorov General Physics Institute of RAS

38, Vavilov str., Moscow, 119991, Russia

${ }^{b}$ Institute for Problems of Materials Science of UAS

3, Krzhyzhanovsky str., 03680 Kiev, Ukraine

${ }^{c}$ Center of Low Temperature Physics, IEP SAS, 04001 Košice, Slovakia

${ }^{d}$ Hahn-Meitner Institute, 100, Glienicker Str., 14109 Berlin, Germany

High precision measurements of magnetoresistance $\Delta \rho / \rho=f(T, H)$ and magnetization $M(T, H)$ were carried out on single crystals of rare-earth dodecaboride $\mathrm{ErB}_{12}$ at temperatures in the interval 1.8-30 $\mathrm{K}$ in magnetic fields up to $70 \mathrm{kOe}$. The high accuracy of the experiments allowed us to perform numerical differentiation and analyze quantitatively the behavior of the derivative $\mathrm{d}(\Delta \rho / \rho) / \mathrm{d} H=f(T, H)$ and of the magnetic susceptibility $\chi(T, H)=\mathrm{d} M / \mathrm{d} H$ in paramagnetic and magnetically ordered (antiferromagnetic, $T_{\mathrm{N}} \approx 6.7 \mathrm{~K}$ and $T_{\mathrm{M}} \approx 5.85 \mathrm{~K}$ ) phases of $\mathrm{ErB}_{12}$. It was shown that negative magnetoresistance anomalies observed in present study in paramagnetic state of $\mathrm{ErB}_{12}$ may be consistently interpreted in the framework of a simple relation between resistivity and magnetization $-\Delta \rho / \rho \sim M^{2}$.

PACS numbers: 72.15.Eb, 75.50.Ee

\section{Introduction}

During the past decade antiferromagnetic (AF) dodecaborides $\mathrm{ReB}_{12}$ containing rare-earth $(\mathrm{Re})$ atoms in the sites of fcc lattice have received renewed attention $[1,2]$. In these compounds the AF-ordering of the ensemble of $\mathrm{Re}^{3+}$ ions' magnetic moments is characterized by small enough Néel temperatures $k_{\mathrm{B}} T_{\mathrm{N}} \leq 1-2 \mathrm{meV}[3]$, and $\mathrm{ReB}_{12}$ were quoted as model systems to study the frustration effects in the fcc antiferromagnets [2].

In the family of $\mathrm{ReB}_{12}$ compounds erbium dodecaboride is the outstanding representative which demonstrates two zero-field magnetic phase transitions at $T_{\mathrm{N}} \approx 6.7 \mathrm{~K}$ and $T_{\mathrm{M}} \approx 5.85 \mathrm{~K}$ in combination with a number of unknown high field magnetic phase transitions and a rather complicated $H-T$ magnetic phase diagram [4]. Additionally, significant spin disorder on a short-range scale ( $\leq 5$ lattice

*corresponding author; e-mail: nes@lt.gpi.ru 
constants) has been established by muon spin rotation ( $\mu$ SR)-spectroscopy studies [5] in this stoichiometric compound where the magnetic Er ions are located on well-defined lattice sites. Moreover, it was shown very recently by $\mu \mathrm{SR}[5]$ and diffuse neutron scattering [6] experiments that in the paramagnetic $(\mathrm{P})$ state well above $T_{\mathrm{N}}$ the magnetic response should be interpreted in terms of short-range ordering, in which the correlation length increases remarkably with the temperature lowering towards $T_{\mathrm{N}}[3,6]$.

To shed more light on the nature of low temperature magnetism and the origin of magnetic interactions in $\mathrm{ErB}_{12}$ in this study we present results of precise magnetoresistance (MR) and magnetization (M) measurements on $\mathrm{ErB}_{12}$, and approach their interpretation in terms of the well-known model of Yosida [7]. Single crystalline $\mathrm{ErB}_{12}$ samples were grown by crucible-free inductive zone melting process in pure argon atmosphere [8]. High accuracy transverse magnetoresistance measurements were performed using the standard DC four-probe method in temperature range $1.8-30 \mathrm{~K}$ (temperature stabilization accuracy $\leq 0.01 \mathrm{~K}$ ) in magnetic fields up to $70 \mathrm{kOe.} \mathrm{A} \mathrm{modernized} \mathrm{LDJ-1500} \mathrm{vibrating} \mathrm{sample} \mathrm{magne-}$ tometer was applied for precise magnetization measurements in magnetic fields up to $60 \mathrm{kOe}$.

\section{Results and discussion}

The resistivity temperature dependences for various magnetic fields are shown in Fig. 1 (current $\boldsymbol{I} \|\langle 110\rangle$, field $\boldsymbol{H} \|\langle 001\rangle$ ). The observed double "hump-like maximum" in $\rho(T)$ corresponds to magnetic phase transitions at $T_{\mathrm{N}} \approx 6.7 \mathrm{~K}$ and $T_{\mathrm{M}} \approx 5.85 \mathrm{~K}$. With increasing magnetic field the resistivity decreases essentially both in the paramagnetic phase ( $\mathrm{P}$ region, $T>T_{\mathrm{N}}$ ) and in the AF phase slightly below $T_{\mathrm{N}}$ (Figs. 1, 2). On the contrary, a remarkably positive magnetoresistance (PMR) is observed at $T<T_{\mathrm{M}}$ (Figs. 1, 2) and this effect is accompanied with a noticeable resistivity hysteresis that can be clearly detected in the range $H<20 \mathrm{kOe}$. It should be also mentioned that in the range $H \leq 40 \mathrm{kOe}$ the negative magnetoresistance (NMR) in the $\mathrm{P}$ region may be described precisely

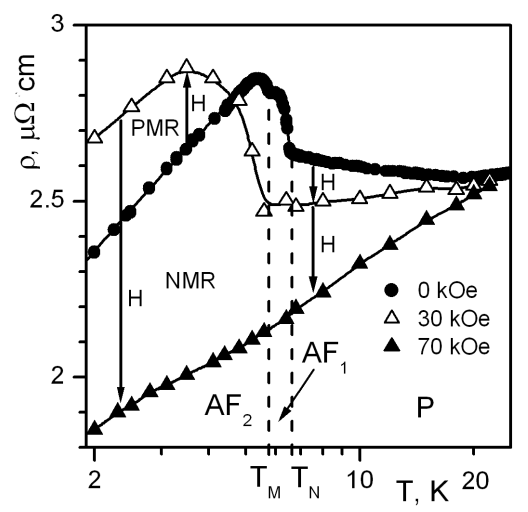

Fig. 1. Temperature dependences of resistivity of $\mathrm{ErB}_{12}$ in different magnetic fields. NMR and PMR represent negative and positive magnetoresistance regions. 

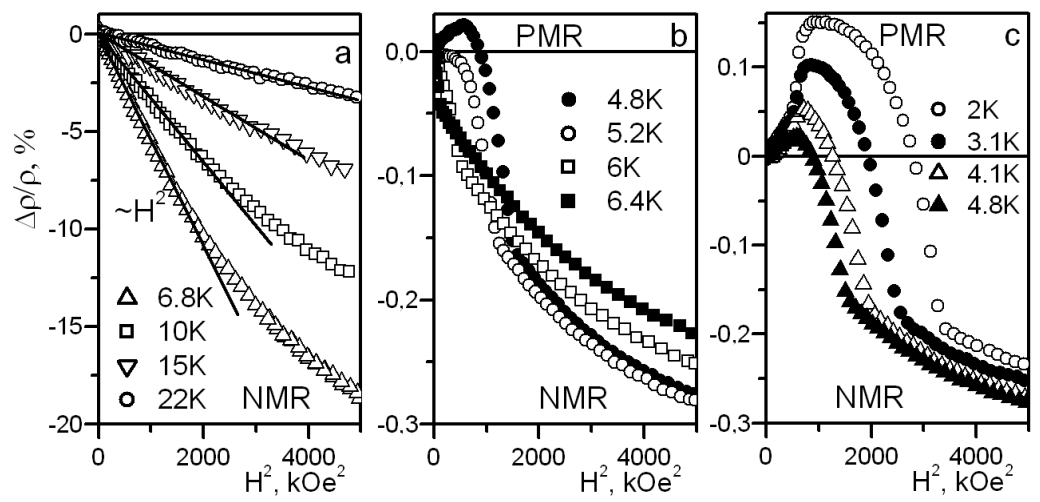

Fig. 2. Magnetic field dependences of magnetoresistance $\Delta \rho / \rho\left(H^{2}\right)$ in paramagnetic (a) and in vicinity of $\mathrm{AF}-\mathrm{P}$ phase transition (b), (c) in $\mathrm{ErB}_{12}$.
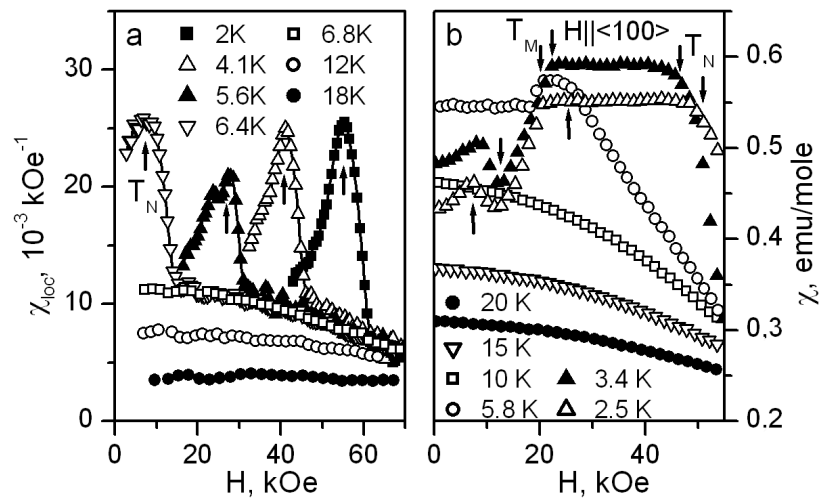

Fig. 3. Magnetic field dependences of local (a) (see text) and bulk (b) magnetic susceptibility $\chi(T, H)=\mathrm{d} M / \mathrm{d} H$ of $\operatorname{ErB}_{12}$ at temperatures in the range $2-20 \mathrm{~K}$.

by the relation $-\Delta \rho / \rho \sim H^{2}$ (Fig. 2), and at high magnetic field a tendency to saturation can be detected on $\Delta \rho / \rho=f(H)$ dependences.

The most appropriate interpretation of the NMR effect in $\mathrm{ErB}_{12}$ can be obtained within the framework of the approach developed by Yosida [7], where a simple relation between magnetoresistance and magnetization $\Delta \rho / \rho \sim M_{\mathrm{loc}}^{2} \sim \chi_{\mathrm{loc}}^{2} H^{2}$ has been predicted. To verify the prediction of [7] the local magnetic susceptibility $\chi_{\text {loc }}(T, H)=[1 / H(\mathrm{~d}(\Delta \rho / \rho) / \mathrm{d} H)]^{1 / 2}$ was deduced directly from $\Delta \rho(H, T)$ data (Fig. 3a) and compared with the bulk susceptibility results $\chi(T, H)=\mathrm{d} M / \mathrm{d} H$ (Fig. 3b) which were obtained by numerical differentiation of the experimental magnetization data. The very similar behavior of $\chi_{\mathrm{loc}}(T, H)$ and $\chi(T, H)$ dependences in paramagnetic state can be considered as an evidence in favor of Yosida's approach [7] to describe the charge transport in $\operatorname{ErB}_{12}$. Moreover, the $\Delta \rho / \rho(T, H)$, $\chi_{\mathrm{loc}}(T, H)$ and $\chi(T, H)$ dependences obtained in this study allow us to analyze the complicated $H-T$ magnetic phase diagram of this compound (see Fig. 4). 


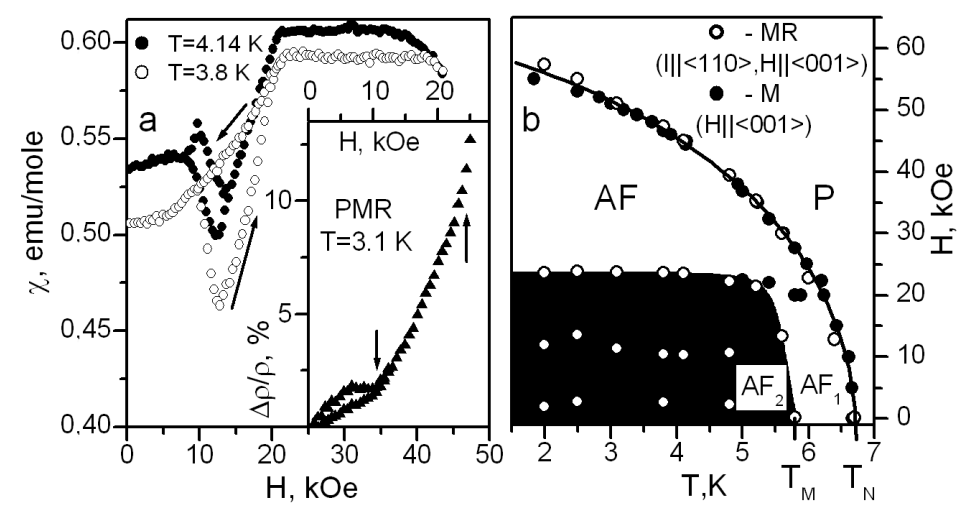

Fig. 4. Magnetic field dependences of bulk magnetic susceptibility $\chi(H)$ (a) and positive magnetoresistance $\Delta \rho / \rho(H)$ (inset) of $\operatorname{ErB}_{12}$. Magnetic $H-T$ phase diagram (b) of $\mathrm{ErB}_{12}$. The hysteresis region is hutched.

The most interesting and unknown feature of the $H-T$ diagram that should be emphasized here is the complicated transformation of $\mathrm{ErB}_{12}$ magnetic structure including magnetic phase transition at about $20 \mathrm{kOe}$ and essential hysteresis effects (see Fig. 4a) on both the resistivity and susceptibility field dependences in the magnetically ordered state of erbium dodecaboride. To understand this state in detail, more investigations of this compound should be undertaken.

\section{Acknowledgments}

Financial supports of INTAS 03-51-3036, VEGA-7054 and RAS Program "Strongly Correlated Electrons" are acknowledged.

\section{References}

[1] A. Czopnik, N. Shitsevalova, A. Krivchikov, V. Pluzhnikov, Yu. Paderno, Y.Onuki, J. Solid State Chem. 177, 507 (2004).

[2] K. Siemensmeyer, K. Habicht, Th. Lonkai, S. Mat'ash, S. Gabani, N. Shitsevalova, E. Wulf, K. Flachbart, J. Low Temp. Phys. 146, 581 (2007).

[3] S. Gabani, I. Bat'ko, K. Flachbart, T. Herrmannsdörfer, R. König, Y. Paderno, N. Shitsevalova, J. Magn. Magn. Mater. 207, 131 (1999).

[4] P. Priputen, K. Flachbart, S. Gabani, S. Gabáni, V. Pavlík, Y. Paderno, N. Shitsevalova, Acta Phys. Slov. 56, 157 (2006).

[5] G.M. Kalvius, D.R. Noakes, N. Marcano, R.WWäppling, F. Iga, T. Takabatake, Physica B 326, 398 (2003).

[6] K. Siemensmeyer, K. Flachbart, S. Gabani, S. Matfas, Y. Paderno, N. Shitsevalova, J. Solid State Chem. 179, 2748 (2006).

[7] K. Yosida, Phys. Rev. 107, 396 (1957).

[8] Yu. Paderno, V. Filippov, N. Shitsevalova, in: Boron-Rich Solids, AIP Conf. Proc., Eds. D. Emin, T.L. Aselage, A.C. Switendick, E.B. Morosin, C.L. Beckel, Vol. 230, Albuquerque 1991, p. 460. 